\title{
Thermal Excitation of Modes in a Non-neutral Plasma
}

\author{
Roy W. Gould
}

California Institute of Technology, Pasadena, CA 91125

\begin{abstract}
We examine theoretically the thermal fluctuations in a non-neutral plasma, as observed on a segmented electrode surrounding the plasma, using the Nyquist theorem. The fluctuation spectrum is peaked at frequencies which correspond to modes of the plasma. Measurement of these fluctuations and of the input admittance of the plasma in the vicinity of one of the mode resonances can be used to give a completely experimental, and nondestructive, measurement of the plasma temperature. Since some of the modes are negative energy modes and exhibit negative absorption due to the plasma rotation, a direct application of the Nyquist theorems will not give correct results for the non-axisymmetric modes. This problem is circumvented by first calculating the fluctuations in a frame rotating with the plasma and transforming to the laboratory frame. This results in a modification of Nyquist's theorem. Measurement issues are discussed.
\end{abstract}

\section{INTRODUCTION}

Recent experimental measurements[1] of the fluctuations in a cylindrical pure electron plasma show that the fluctuation spectrum is peaked at frequencies of the Trivelpiece-Gould modes[2] and that they are thermal in origin. Measurements are made by observing the frequency spectrum of the fluctuating signal on a "patch" electrode which is part of the conducting cylinder which surrounds the plasma. We show the situation schematically in Figure 1. We show the field lines of a few

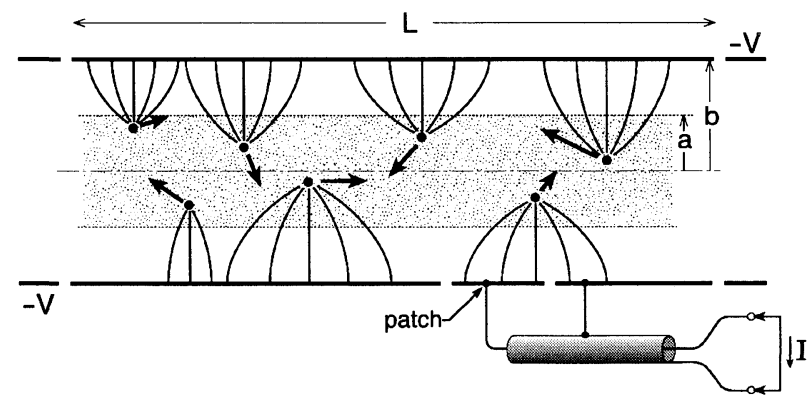

FIGURE 1. Charged particles in a cylindrical conducting cylinder. Field lines to the wall are indicated and arrows indicate the particle velocity. Fluctuations of the charge on patch result in a current $I(\omega)$ in the external circuit.

CP606, Non-Neutral Plasma Physics IV, edited by F. Anderegg et al. (c) 2002 American Institute of Physics 0-7354-0050-4/02/\$19.00 263 
selected particles. They terminate on charges which reside on the conducting wall at $r=b$. As the particles move, these wall charges change so that there are fluctuating charges and currents induced on the wall. We show a small patch used to measure the fluctuations. We want to find the fluctuating charge on the patch or, alternatively, the fluctuating (displacement) current which flows to the patch.

There are various methods by which fluctuations can be calculated. In thermal equilibrium, the principle of detailed balance says that emission and absorption must be balanced, so that one can obtain the emission from a knowledge of the absorption and the temperature. Nyquist's Theorem[3] is a well-known application to electrical circuits which relates thermal fluctuations to the dissipative part of the admittance or impedance functions of an electrical circuit. This theorem was later generalized to Hamiltonian systems by Callen and Welton[4] and applied to a wide class of dynamical systems. It is called the generalized Nyquist theorem, or the fluctuationdissipation theorem. These methods require a knowledge of the input admittance of the patch. In plasmas, fluctuating quantities can also be calculated from two-particle distribution functions, or using Rostoker's dressed test particle approach[5]. The latter approach is not limited to systems in thermodynamic equilibrium (although they must be stable) but is harder to apply except in the most simple situations (e.g. spatially uniform plasmas).

This paper is divided into two parts: a discussion of the application of Nyquist's theorem to the axisymmetric $(m=0)$ modes, then a discussion of the nonaxisymmetric $(m \neq 0)$ modes, some of which are negative energy modes to which Nyquist theorem cannot be applied directly. Instead, we calculate the fluctuations in the rotating frame using Nyquist's theorem, where all modes are positive energy modes. We then transform this result to the laboratory frame. This leads to a modification of the Nyquist result, essentially an extension of Nyquist's theorem to rotating systems.

\section{NYQUIST'S THEOREM}

Nyquist's theorem relates the mean-square fluctuations to the real part of the admittance of electric circuit, $Y(\omega)=I(\omega) / V(\omega)$. As seen from the coaxial connector in Figure 1, we can view the plasma as an electric circuit which has an admittance, $Y_{p}(\omega)$, which describes the linear response of the plasma. We have previously discussed[6] the properties of $Y_{p}(\omega)$ in connection with the transfer of angular momentum and energy to a plasma through the excitation of nonaxisymmetric Trivelpiece-Gould modes. This function has simple poles at each of the mode frequencies. $Y_{p}(\omega)$ is also a quantity which can be measured experimentally. It reflects the properties of the plasma and we shall assume that it is at the temperature of the plasma, $T_{p}$. Accordingly, we have the equivalent electrical circuit of Figure 2, 


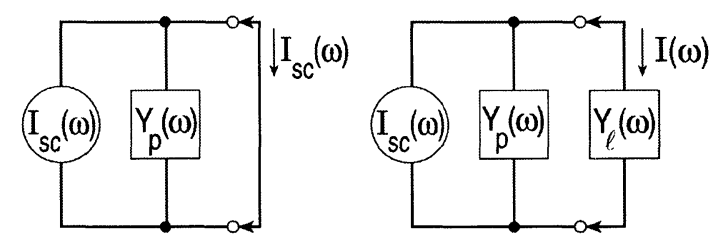

FIGURE 2. (a) Norton Equivalent Circuit with a short circuit,

(b) Norton Equivalent Circuit with a load admittance, $Y_{l}$

in which the effect of the thermal fluctuations of the circuit are represented by a current generator with mean square current $\left|I_{s c}^{2}(\omega)\right|$ in frequency range $d \omega$

$$
\left|I_{s c}^{2}(\omega)\right|=\frac{2}{\pi} \kappa T_{p} \operatorname{Re}\left\{Y_{p}(\omega)\right\} d \omega
$$

in parallel with $Y_{p}(\omega)$, as illustrated in Figure 2.

Near one of the mode frequencies where the plasma can be resonantly excited, $Y_{p}(\omega)$ has a simple pole at the mode frequency so that

$$
Y_{p}(\omega) \simeq \frac{R_{\text {res }}}{-i\left(\omega-\omega_{\text {res }}\right)+\gamma_{\text {res }}},
$$

where $\omega_{\text {res }}$ and $\gamma_{\text {res }}$ are the frequency and line width of the mode[6]. $R_{\text {res }}$ is the residue at the pole and depends upon the sector position and dimensions, as discussed later in the paper. Note that the admittance is real and equal to $R_{\text {res }} / \gamma_{r e s}$ at line center. Furthermore, $\left|I_{s c}^{2}(\omega)\right|$ has a Lorentzian lineshape.

The basic idea is to measure both the fluctuation spectrum, e.g. the mean-square current flowing in the load admittance, and the input admittance and then to use Eq. 1 to determine the plasma temperature. A very important point here is that the external load affects the measurement and must be properly taken into account in interpreting the measurements. Note that the current flowing through the load, $I(\omega)$, is generally less than the short-circuit current $I_{s c}(\omega)$ by the factor $Y_{l}(\omega) /\left[Y_{l}(\omega)+Y_{p}(\omega)\right]$ and the voltage across the load is $V(\omega)=I(\omega) / Y_{l}(\omega)$. The external load will, in general, introduce additional mode damping and make an additive contribution to $\gamma_{r e s}$, thus increasing the linewidth. Since the load is not, in general, at the same temperature as the plasma it may also cool the mode slightly. The mode is thermally excited by the particles at $T_{p}$ but also cooled by an external load at a lower temperature, $T_{l}$. The mode temperature is determined by a competition between these processes. Furthermore, if not all parts of the system are at temperature $T_{p}$, then the measured temperature will be a weighted average temperature, weighted according to the dissipation of the various parts. Thus the copper electrodes, which have very little dissipation, will not affect the measured temperature significantly. If there are radial temperature gradient (electrons equilibrate along field lines very quickly but not necessarily across field lines) we expect observed temperature to be an average 
temperature, weighted according to where the dissipation occurs. Since the $m=0$ and $m=2$ mode fields have different radial dependences, one might be able to determine whether a radial temperature gradient exists from measurements using two different modes.

\section{NON-AXISYMMETRIC MODES AND NEGATIVE ABSORPTION}

In thermal equilibrium emission is related to absorption, and fluctuations to dissipation. The axisymmetric modes are all positive energy modes and the Nyquist theorem works fine. However some of the non-axisymmetric modes are negative energy modes, i.e. their excitation releases energy from the plasma and they exhibit negative dissipation. Recall that the $m=1$ diocotron mode can be destabilized by attaching a resistor to the patch[7], and that the damping of the $m=2$ diocotron mode is reduced by attaching a resistor to the patch[8]. It is the modes whose azimuthal phase velocity are less than the rotation angular velocity, $0<\omega / m<\omega_{\text {rot }}$, which we shall refer to as the slow modes, which are negative energy modes. Their excitation releases some of the energy of rotation. The real part of the patch input admittance associated with modes in this frequency range is negative. Nyquist's theorem (and the fluctuation-dissipation theorem) can not be applied to such a situation. Yet we expect the plasma to be in thermal equilibrium if these modes are not destabilized.

The reason for this paradox is the nature of the equilibrium. In making the case for thermal equilibrium, it is generally assumed that the walls are smooth, the system is axisymmetric, and angular momentum and energy are constants of the motion[9]. The equilibrium which results is very special, the plasma is rotating as a whole and has angular momentum. For some purposes, including the discussion of thermal fluctuations, it is more appropriate to view the plasma in the rotating frame, in which the plasma appears stationary. In this frame the plasma has no negative energy modes. We expect that the dynamics will be the same in either frame, except for the coordinate transformation. The effect of transforming to the rotating frame introduces a uniform rigid neutralizing background charge. This situation is frequently referred to as the one-component-plasma (OCP).

We can apply the Nyquist theorem (or fluctuation-dissipation theorem) in the rotating (primed) frame, i.e. to the OCP. For this purpose we will not consider a patch electrode, but will instead express the potentials and electric field at the wall $(r=b)$ in terms of their Fourier components (with $k=n \pi / L)$,

$$
\begin{aligned}
\phi^{\prime} & =\sum_{k, m} \phi_{k m}^{\prime} e^{i k z+i m \theta^{\prime}-i \omega^{\prime} t} \\
E_{r}^{\prime} & =\sum_{k, m} E_{r k m}^{\prime} e^{i k z+i m \theta^{\prime}-i \omega^{\prime} t} .
\end{aligned}
$$

We calculate the fluctuations of the various Fourier components in the rotating frame and then transform that result to the laboratory (unprimed) frame. The 
frequencies of the fluctuations will be Doppler shifted: $\omega^{\prime}=\omega-m \omega_{r}$ or $\omega=\omega^{\prime}+m \omega_{r}$, where $\omega_{r}$ is the rotational angular velocity. The mode frequencies $\omega_{m n p}$ are also Doppler shifted by the rotation so that $\omega_{m n p}=\omega_{m n p}^{\prime}+m \omega_{r}$.

We imagine that a small potential (one Fourier component) is applied at $r=b$ and that this produces a corresponding Fourier component of the radial electric field at $r=b$ which is proportional to the applied potential. We define the linear response function, or susceptibility $\chi_{k m}^{\prime}\left(\omega^{\prime}\right)$, of the plasma[6] as the ratio of the radial electric field produced to the potential which causes it, i.e.

$$
E_{r k m}^{\prime}=-\chi_{k m}^{\prime}\left(\omega^{\prime}\right) \frac{\phi_{k m}^{\prime}}{b}
$$

To apply Nyquist theorem, we need a current to go along with the potential. For this purpose we define a displacement current at the wall $I_{k m}^{\prime}=-i \omega^{\prime} \epsilon_{o} S E_{r k m}^{\prime}$. The complex power delivered to the plasma is

$$
P=\frac{1}{2} \int_{S} i \omega^{\prime} \epsilon_{o} E_{r}^{\prime} \phi^{\prime *} d S=\frac{1}{2} i \omega^{\prime} \epsilon_{o} S \sum_{k^{\prime} m^{\prime}} E_{r k m}^{\prime} \phi_{k m}^{\prime *}=\frac{1}{2} \sum_{k m} I_{k m}^{\prime} \phi_{k m}^{\prime *}
$$

where $S=2 \pi b L$ is the total area of the cylinder, $I_{k m}^{\prime}$ is the current, and * denotes the complex conjugate. Because $E_{r k m}^{\prime}$ is proportional to $\phi_{k m}^{\prime}, I_{k m}^{\prime}$ is also proportional to $\phi_{k m}^{\prime}$. Thus

$$
I_{k m}^{\prime}=\left[\frac{-i \omega^{\prime} \epsilon_{o} S}{b} \chi_{k m}^{\prime}\right] \phi_{k m}^{\prime}=Y_{k m}^{\prime} \phi_{k m}^{\prime}
$$

$Y_{k m}^{\prime}$ is the admittance of one Fourier component which we shall use in Nyquist's theorem to determine the fluctuation in electric field at the wall, and the corresponding fluctuating current and wall surface charge density in frequency range $d \omega^{\prime}$ are:

$$
\begin{gathered}
\left|I_{k m}\left(\omega^{\prime}\right)\right|^{2}=\frac{2}{\pi} \kappa T \operatorname{Re}\left\{Y_{k m}^{\prime}\left(\omega^{\prime}\right)\right\} d \omega^{\prime}, \\
\left|\sigma_{k m}\left(\omega^{\prime}\right)\right|^{2}=\frac{1}{\omega^{\prime 2} S^{2}}\left|I_{k m}\left(\omega^{\prime}\right)\right|^{2}=\frac{2}{\pi} \kappa T \frac{\epsilon_{o}}{S b} \operatorname{Im}\left\{\frac{\chi_{k m}^{\prime}\left(\omega^{\prime}\right)}{\omega^{\prime}}\right\} d \omega^{\prime} .
\end{gathered}
$$

$\operatorname{Im}\left\{\frac{\chi_{k m}\left(\omega^{\prime}\right)}{\omega^{\prime}}\right\}$ is always positive because the system is in equilibrium in the rotating frame and therefore stable. The fluctuations are the same in both frames, except for Doppler shift of frequencies $\omega^{\prime}=\omega-m \omega_{r}$. The linear response function is the same in both frames except for Doppler shift of frequencies, i.e. $\chi_{k m}(\omega)=\chi_{k m}^{\prime}\left(\omega^{\prime}\right)$. Finally, the fluctuating wall charge in the laboratory frame is

$$
\left|\sigma_{k m}(\omega)\right|^{2}=\frac{2}{\pi} k T \frac{\epsilon_{o}}{S b} \operatorname{Im}\left\{\frac{\chi_{k m}^{\prime}\left(\omega-m \omega_{r}\right)}{\omega-m \omega_{r}}\right\} d \omega .
$$

From the Fourier components of the fluctuating charge we can calculate the fluctuating patch current when it is short-circuited 


$$
I_{s c}=-i \omega \int_{S_{p}} \sigma d S=-i \omega S_{p} \sum_{k m} F_{k m} \sigma_{k m},
$$

where $S_{p}$ is the patch area and $F_{k m}$ is a patch shape factor $(\leq 1)$. The mean square fluctuating current, $\left|I_{s c}(\omega)\right|^{2}$, is therefore

$$
\left|I_{s c}(\omega)\right|^{2}=\frac{2}{\pi} \kappa T \frac{\omega \epsilon_{o} S_{p}^{2}}{S b} \sum_{k m}\left|F_{k m}\right|^{2} \operatorname{Im}\left\{\frac{\omega}{\omega-m \omega_{r}} \chi_{k m}^{\prime}\left(\omega-m \omega_{r}\right)\right\} d \omega
$$

Direct calculation of the patch admittance in the lab frame gives

$$
Y_{p}(\omega)=\frac{\omega \epsilon_{S} S_{p}^{2}}{S b} \sum_{k m}\left|F_{k m}\right|^{2} \operatorname{Im}\left\{\chi_{k m}(\omega)\right\}
$$

Patch fluctuations are no longer simply related to patch admittance. However, Equation (6) is much simpler when only one azimuthal wave number contributes significantly to the admittance (for example, near the resonance of a mode). In this case

$$
\left|I_{s c}(\omega)\right|^{2}=\frac{2}{\pi} \kappa T \operatorname{Re}\left\{\frac{\omega}{\omega-m \omega_{r}} Y_{p}(\omega)\right\} d \omega \quad .
$$

Thus even when $\operatorname{Re}\left\{Y_{p}(\omega)\right\}<0$ (for potentially unstable modes) we can obtain the fluctuation level in thermal equilibrium. This result is very similar to the Nyquist result, the difference being that the factor $\frac{\omega}{\omega-m \omega_{r}}$ appears. This factor is negative whenever $\operatorname{Re}\left\{Y_{p}(\omega)\right\}$ is negative, so that the product is always positive. Notice however that this factor is important even for the positive energy non-axisymmetric modes (when both factors are positive). It reflects the effect of rotation on the magnitude of the fluctuation spectrum.

\section{DISCUSSION}

To determine the plasma temperature, measure the fluctuating voltage $\left|V^{2}(\omega)\right|$ across the load admittance near one of the mode resonances with a load $Y_{l}(\omega)$ connected to the patch. Also measure the input admittance of the plasma $Y_{p}(\omega)$ at very low signal level so as not to drive the plasma mode into the nonlinear regime. This measurement can be done by measuring the complex reflection coefficient of the plasma with a $50 \Omega$ directional coupler, converting the measured reflection coefficient, $r(\omega)$, to the complex admittance using ${ }^{1} Y(\omega) / Y_{o}=[1-r(\omega)] /[1+r(\omega)]$, then using the expression

$$
\left|V^{2}(\omega)\right| \approx 4 k T_{p} \operatorname{Re}\left\{\frac{\omega}{\omega-m \omega_{r}} Y_{p}(\omega)\right\} d \omega / \mid Y_{l}\left(\omega+\left.Y_{p}(\omega)\right|^{2}\right.
$$

1. The loci of both $Y(\omega)$ and $r(\omega)$ versus $\omega$ are circles in the complex plane. This property can be useful in fitting experimental data. 
to obtain the plasma temperature $T_{p}$. This assumes that one mode dominates the fluctuation spectrum and the plasma admittance. For the $m=0$ modes the new factor $\frac{\omega}{\omega-m \omega_{r}}$ is unity, but for all non-axisymmetric modes this factor must be taken into account.

In making measurements it is important that the load admittance not destabilize any of the negative energy modes. An axisymmetric patch does not couple to the negative energy modes so this is not an issue. However if the patch is not axisymmetric, the real part of the load admittance should be very small at the frequencies which might lead to destabilization of any negative energy modes. There must be no, or very little, growth of these modes prior to or during a measurement. This can be accomplished with a capacitive load, which will only cause a small frequency shift of the mode.

\section{CONCLUSIONS}

The thermal equilibrium of a non-neutral plasma is an exceptional kind of equilibrium. It has angular momentum and energy due to its rotation and it is not in thermal equilibrium with the outside world. However, it is in thermal equilibrium in a rotating frame when there are no non-axisymmetric perturbations so that the system has constant angular momentum. A patch electrode which produces only axisymmetric fields does not break the angular momentum constraint and does not destroy the equilibrium. Such a patch electrode can be use to weakly excite the axisymmetric modes of the plasma and to measure the thermal fluctuations of these modes without destroying the thermal equilibrium of the plasma. This makes possible a non-destructive, purely experimental, measurement of the plasma temperature using the Nyquist theory of fluctuations associated with these modes in thermal equilbrium.

On the other hand, the slow non-axisymmetric modes have negative energy and exhibit negative resistance and negative absorption. Nyquist's theorem of fluctuations can not be applied directly to these modes because the real part of their input admittance is negative. However, we have shown here that it is still possible to relate the fluctuations associated with non-axisymmetric modes to the real part of the input admittance, even when the latter is negative! In doing so, we have derived a modified Nyquist theorem for rotating equilibria. In this new result their appears an additional factor

$$
\omega /\left(\omega-m \omega_{r}\right),
$$

which is negative for the negative energy slow modes for which the real part of the patch impedance is negative. For the axisymmetric modes, this new factor is unity and we recover the usual Nyquist result.

We obtained this result by calculating the fluctuations in the frame rotating with the plasma, where the plasma is in thermal equilibrium and then transforming the result to the laboratory frame. In the rotating frame all modes are stable and none are 
negative energy modes. Although the results of this paper were derived for cylindrical plasmas, it is clear they apply as well to spheroidal plasmas[10].

Finally, it should be possible to observe the fluctuations associated with nonaxisymmetric modes, and to use them to determine the plasma temperature. However, in dealing with non-axisymmetric patches and modes, it is important that the load admittance connected to the patch not have a significant real part, else a negative energy mode may be destabilized and slowly grow. This can be achieved by using a capacitive load, whose effect is to shift the mode frequency very slightly.

\section{ACKNOWLEDGEMENT}

The author is grateful to F. Anderegg for communicating the experimental results and to members of the U.C.S.D group for stimulating discussions.

\section{REFERENCES}

[1] F. Anderegg, N. Shiga, J. R. Danielson, C. F. Driscoll, D. E. Dubin, and R. W. Gould, "Thermal Excitation of Trivelpiece-Gould Modes in a Pure Electron Plasma" in Non-Neutral Plasma Physics IV (these proceedings), edited by F. Anderegg, L. Schweikhard, and C. F. Driscoll.

[2] A. W. Trivelpiece and R. W. Gould, J. Appl. Phys. 30, 1784 (1959), S. A. Prasad and T. M. O'Neil, Phys. Fluids 22, 278 (1979).

[3] H. Nyquist, Phys. Rev. 32, 110 (1928).

[4] H. B. Callen and T. A. Welton, Phys. Rev. 83, 34 (1951).

[5] N. Rostoker, Nuclear Fusion, 1, 101 (1961)

[6] R. W. Gould, "Wave Angular Momentum in Non-neutral Plasmas", Non-Neutral Plasma Physics III, Am. Inst. Phys. p 170 (1999).

[7] W. D. White, J. H. Malmberg and C. F. Driscoll, Phys. Rev. Lett. 49,1822 (1982).

[8] N. S. Pillai and R. W. Gould, Phys. Rev. Lett. 73, 2849 (1994).

[9] T. M. O'Neil and D. H. E. Dubin, Phys. Plasmas 5, 2163 (1998).

[10] D. H. E. Dubin, Phys. Rev. Lett. 66, 2076 (1991). 